STRUCTURAL BIOLOGY COMMUNICATIONS

ISSN 2053-230X

\section{Acta Crystallographica Section F - another home for cryo-electron microscopy contributions}

\author{
Alok K. Mitra ${ }^{\mathrm{a} *}$ and Mark van Raaij ${ }^{\mathrm{b} *}$

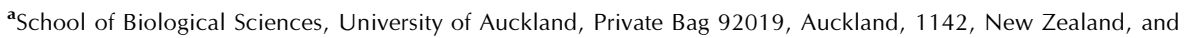 \\ ${ }^{\mathbf{b}}$ Department of Molecular Structure, Centro Nacional de Biotecnologia, Consejo Superior de Investigaciones Cientificas, \\ E-28049 Madrid, Spain. *Correspondence e-mail: a.mitra@auckland.ac.nz, mjvanraaij@cnb.csic.es
}

We are pleased to present in this issue a collection of invited contributions on current state-of-the-art cryo-electron microscopy (cryo-EM). These contributions are to acknowledge and reflect the transformational changes in the discipline of modern cryo-EM in the past few years that have led to a remarkable number of high-resolution cryo-EM structures of isolated biological macromolecules being solved for the first time. In this context, 2017 was a particularly notable year, with the Nobel prize in Chemistry awarded jointly to Jacques Dubochet, Richard Henderson and Joachim Frank for the development of cryo-EM (Callaway, 2017), which highlighted the current progress.

The meteoric progress of cryo-EM has been enabled by the availability of direct detectors, high-throughput ultra-stable $300 \mathrm{kV}$ electron microscopes and clever imageprocessing algorithms exploiting powerful computer hardware. These advances have facilitated the generation of density maps for de novo semi-automated atomic modelling (Terwilliger et al., 2018), often in just a few days (Kimanius et al., 2016). Large macromolecular complexes $(>\mathrm{MDa})$, requiring handling of very large volumes of image data that was not manageable until only a few years ago, are being increasingly investigated. In addition, attractive aspects, such as the requirement for relatively small amounts of a sample that does not need to be purified to biochemical homogeneity and the fact that no crystals are required, have contributed significantly to cryo-EM's current popularity in structural biology.

The Topical Review (Mitra, 2019) published in this collection provides an historical background to advances in methods development in transmission electron microscopy, and how it has evolved into the discipline of modern cryo-EM as applied to biological macromolecules. It discusses the present state of the art and indicates the likely direction of future developments.

Three Research Communications discuss important issues in contemporary highresolution cryo-EM image processing. In cryo-EM, single-particle analysis (SPA) is based on averaging low-contrast (low signal-to-noise ratio) 2D projection images to ameliorate the effect of noise and thereby enhance the resolution of the 3D reconstruction. Radermacher and Ruiz discuss the influence of noise in such cross-correlation analysis, and indicate an optimum method to 'correct' for the major effect of noise in scrutinizing possible bias in image alignments and classification (Radermacher \& Ruiz, 2019).

With regard to the exact definition of resolution, it should be noted here that there is no rock-solid consensus in cryo-EM SPA. Nevertheless, an interesting question on the relationship of image-pool size and achievable resolution is addressed by Heymann, who surmises that beyond reaching a nominal resolution (e.g. near atomic, $\sim 4 \AA$ ) limited by factors such as sample variability, attempts to achieve atomic resolution may require an order-of-magnitude enhancement of the image pool (Heymann, 2019).

The 2D images recorded in SPA capture any discreet or continuous conformational heterogeneity that can be at the core of biological function. Unless separated into homogeneous pools, image heterogeneity can compromise the resolution achieved. On this important theme, Sorzano and colleagues review approaches using theoretical angles of dynamics and modelling that are being applied in the field to analyze heterogeneity (Sorzano et al., 2019). Such studies could be supplemented by incorporating direct analysis of experimental images, as proposed recently for instance (Frank, 2018). 
Overall, it is no understatement to say that the rise in cryoEM has indeed led to exciting times in the field of structural biology. Not only for microscopists specializing in cryo-EM but also for life scientists in general. However, the so-called resolution revolution (Kühlbrandt, 2014) notwithstanding, notable success also engenders notable circumspection. Issues, for instance, in specimen preparation and imaging such as protein denaturation at the air-water interface and the challenge posed by the major cohort of typical proteins of 'small' mass $(<100 \mathrm{kDa})$ constitute some of the bottlenecks that hinder further progress in cryo-EM that need robust userfriendly solutions.

Here at Acta Crystallographica $F$, we would like to participate in showcasing the growth of cryo-EM, particularly as it complements the work of the current generation of X-ray crystallographers and nuclear magnetic resonance spectroscopists from the two other existing pillars of structural biology. The number of cryo-EM manuscripts submitted is growing in leaps and bounds, and we would like our journal to be a discerning forum for speedy publication of short contributions of notable impact in the field.

\section{References}

Callaway, E. (2017). Nature, 550, 167.

Frank, J. (2018). Biochemistry, 57, 888.

Heymann, B. (2019). Acta Cryst. F75, 33-44.

Kimanius, D., Forsberg, B., Scheres, S. J. W. \& Lindahl, E. (2016). eLife, 5, e18722.

Kühlbrandt, W. (2014). Science, 343, 1443-1444.

Mitra, A. K. (2019). Acta Cryst. F75, 3-11.

Radermacher, M. \& Ruiz, T. (2019). Acta Cryst. F75, 12-18.

Sorzano, C. O. S., Jiménez, A., Mota, J., Vilas, J. L., Maluenda, D., Martínez, M., Ramírez-Aportela, E., Majtner, T., Segura, J., Sánchez-García, R., del Caño, L., Conesa, P., Melero, R., Jonic, S., Vargas, J., Cazals, F., Freyberg, Z., Krieger, J., Bahar, I., Marabini, R. \& Carazo, J. M. (2019). Acta Cryst. F75, 19-32.

Terwilliger, T. C., Adams, P. D., Afonine, P. V. \& Sobolev, O. A. (2018). Nat. Methods, 15, 905-908. 